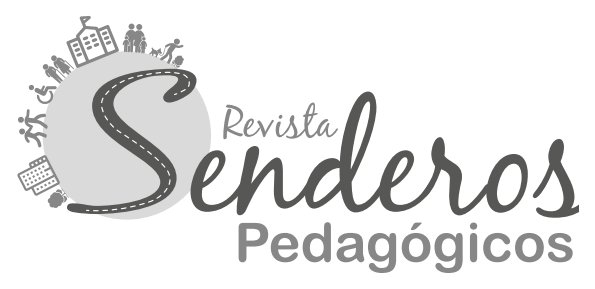

\title{
La metáfora: una aliada del aprendizaje ${ }^{1}$
}

\author{
Metaphor: an ally of learning
}

\author{
Autor: \\ Deiler Hernando Molina Rodelo ${ }^{2}$ \\ https://orcid.org/0000-0003-0996-5880 \\ Recibido: 30/05/2021 \\ Aprobado: 30/10/2021
}

Artículo de reflexión.

2 Magíster en Lingüística, Universidad de Antioquia; Licenciado en Educación Básica con énfasis en Humanidades y Lengua Castellana y docente de cátedra del Tecnológico de Antioquia - Institución Universitaria. deilerm@hotmail.com.

\section{Resumen:}

La capacidad de comunicarnos no deja de asombrarnos, a pesar de ser algo tan usual en nuestras vidas. Siendo algo tan frecuente, nos ha permitido generar y transmitir los más grandes conocimientos conocidos de los cuales, muchos, se han expresado en forma de metáforas. En este artículo se hablará de algunas metáforas que se dan en el ámbito cotidiano y pedagógico, de la metáfora como herramienta cognitiva, de la categorización y la integración conceptual con el fin de reconocer la metáfora; uno de los recursos poéticos, comunicativos y cognitivos más mencionados, como un elemento inherente en la actividad de la enseñanza y el aprendizaje.

Palabras clave: metáfora, metáfora cognitiva, pedagogía, herramienta cognitiva, aprendizaje.
Abstract:
The ability to communicate never ceases to amaze us, despite of being something so usual in our everyday lives. As 
frequent as it is, it has led us to generate and transmit the greatest discoveries and knowledge, of which, many, have been expressed as metaphors. This article talks about some metaphors that occur in the daily and pedagogical field, metaphor as a cognitive tool, categorization, and conceptual blending to recognize the metaphor, one of the most known poetics, communicative and cognitive resources as inherent element of the learning process.

Keywords: metaphor, cognitive metaphor, pedagogy, cognitive tool, learning.

\section{Introducción}

Quizá la historia universal es la historia de la diversa entonación de algunas metáforas Jorge Luis Borges

La capacidad de comunicarnos no deja de asombrarnos, a pesar de ser algo tan usual en nuestras vidas. La comunicación verbal es una habilidad tan común, que rara vez un individuo se detiene a pensar en cómo es posible esta interacción, y cómo por medio de unos sonidos precisamente articulados puede expresar los temores que paralizan sus acciones, realizar peticiones a su interlocutor o discutir y sostener sus ideas frente a los otros. Resulta imposible imaginar un día en el que alguien pueda estar sin comunicarse con otro por medio de las palabras o que, en la soledad de su habitáculo, no dialogue consigo mismo por medio de frases internas.

Pero no solo usamos las palabras en la interacción diaria para mantenernos vivos o crear amores y amistades, sino que también los más altos conocimientos que hemos logrado como humanidad se han transmitido, discutido y transformado gracias a ellas. Así, la comunicación verbal ha sido una parte fundamental para la construcción y modelación de las ideas humanas que nos han llevado a formar la sociedad como la conocemos actualmente en términos tecnológicos, científicos, pedagógicos, filosóficos, políticos y artísticos.

Es evidente que las palabras aisladas, las que llamamos sustantivos, verbos o preposiciones, por sí solas no han generado lo mencionado; es a través de los recursos retóricos, argumentativos y sintácticos que podemos activar su verdadero poder. Las frases que se construyen utilizando las herramientas cognitivas que nos caracterizan como humanos son las que llevan consigo todo el potencial de descubrir las verdades prácticas del mundo - de las cuales, muchas se han expresado en forma de metáforas- y modificar nuestras formas de interactuar con la materia y con el entorno social. 
Generalmente, estas interacciones sociales se dan en los espacios de formación académica que permiten a jóvenes y adultos preocuparse por los temas relacionados con las ciencias y las artes. Puesto que la discusión de los conocimientos, de las posturas ideológicas y filosóficas, y la búsqueda de una gran cantidad de objetivos humanos, tanto individuales como colectivos, se hace formalmente en las salas educativas y laboratorios, y está motivada por pedagogos, es natural que también se centre la atención en la actividad que estos realizan y en la manera que construyen su discurso; particularmente en el uso que se hace de las metáforas en esta actividad.

Así pues, es sobre las columnas del pensamiento, la lengua y la pedagogía que alzaremos este texto en el que se pretende reconocer la metáfora, uno de los recursos comunicativos y cognitivos más conocidos a nivel general, como un elemento inherente en la actividad de la enseñanza y el aprendizaje.

\section{La metáfora: más que palabras}

La metáfora ha sido reconocida desde la antigua Grecia como un recurso de la lengua que nace de las mentes en las que florece el arte poético. Poetas y oradores pronunciaban las metáforas con el fin de embellecer sus discursos y darle al habla un carácter elevado y digno de los más altos reconocimientos. Su función estética y ornamental ha mantenido la concepción pública de la metáfora como un mero asunto de la literatura y de la lengua.

Aristóteles definió la metáfora como la traslación del nombre de un objeto a otro que comparte con el primero una relación de semejanza. El significado metafórico se diferencia del literal por la traslación que se hace de algún nombre. Por ejemplo, cuando utilizamos el nombre original de una acción para referirnos a ella, hablamos en sentido literal; cuando el mismo nombre se traslada a otro objeto o verbo hablamos en sentido figurado o metafórico:

- Las plantas florecieron en el jardín.

- Las palabras florecieron en sus labios.

Por otra parte, la metáfora no solo aparece en los discursos literarios. Hace más de dos mil años, Cicerón ya argumentaba que había una metáfora rústica; la metáfora usada por los hombres del campo, quienes, no pudiendo encontrar una mejor manera de expresar la condición de sus cosechas, utilizaban expresiones como:

- Las mieses están alegres.

- Los campos tienen sed 3 .

3 De aquí en adelante, se utilizarán las cursivas para llamar la atención sobre el significado metafórico de alguna expresión. 
Este tipo de expresiones surge por la necesidad de comunicar algo y no por la búsqueda estética. Tenemos entonces, dos tipos de metáforas: las que se usan en los discursos literarios o en la retórica, y las que son usadas por la población general. Las metáforas literarias surgen por la voluntad poética del artista; las rústicas, involuntariamente, por la necesidad de expresión.

Así mismo, en el habla actual y cotidiana, se encuentra una gran cantidad de expresiones metafóricas que fácilmente pasan desapercibidas, pero que, al llamarse la atención sobre ellas, dan cuenta de la valiosa productividad de la metáfora por fuera del entorno literario. Por ejemplo, hablamos de la navegación informática, el hundimiento de los proyectos políticos, las luchas contra la corrupción, los combates contra la publicidad engañosa, los volúmenes altos de los sonidos, la alta definición de las pantallas, etcétera. Aunque normalmente no percibamos estas expresiones como ejemplos evidentes de metáfora, sí es posible reconocer ahora que no hay un uso literal del significado de las palabras en cursiva, sino una traslación que, difícilmente, podría llamarse poética.

\section{La metáfora para conocer nuevas cosas}

En 1917, Félix Restrepo, sacerdote, erudito clásico y pedagogo colombiano, escribe el primer diseño de semántica general de la lengua española. En él, dedica un capítulo a la metáfora, en el que afirma que para nombrar las nuevas cosas que se construyen en el mundo "[...] con mucha frecuencia se aplica a los nuevos objetos el nombre de otro objeto conocido que tiene alguna relación con el que se trata de nombrar" (p. 75). Distinguía entre las metáforas eruditas y populares, estas últimas referidas a las que surgen en el habla de los hombres del pueblo, y es en ellas que encuentra que la motivación para crearlas es la comprensión clara de un nuevo concepto que aún no tiene nombre, o este es complejo. Por ejemplo, la cabeza de un clavo, las patas de una mesa, el ariete militar o la grúa $^{5}$ utilizada en la construcción.

Pero esto no ocurre solo para nombrar cosas que inventamos o construimos, sino también para darle nombre a los nuevos descubrimientos científicos que hacemos en el mundo. Aguilar y Arroyo (2008) realizan una investigación sobre las metáforas en el ámbito científico y argumentan que desde que Aristóteles le dio un nombre a este recurso, ha sido muy útil para la ciencia y así, cuando el investigador descubre un fenómeno nuevo, es decir, cuando forma

\footnotetext{
4 Se forma con la palabra latina <aries-etis> que significa carnero. Félix restrepo explica que "así se llamó una máquina de guerra romana, porque la parte que golpeaba los muros tenía forma de cabeza de carnero.” (p. 76). 5 Del latín <Grus> que significa grulla. A esta máquina se le llama así por el parecido con esta ave.
} 
un nuevo concepto, necesita darle un nombre. Como un concepto nuevo no significaría nada para los demás, tiene que recurrir al repertorio del lenguaje ya usado, donde la palabra se encuentra ya relacionada con un significado. A fin de hacerse entender, elige la palabra cuyo significado tenga alguna semejanza con la nueva significación. De esta manera, el término adquiere la nueva significación por medio de la antigua, sin abandonarla. Esto es la metáfora (p. 52).

En su artículo, aparecen diversas metáforas utilizadas en libros de texto, es decir, en libros usados para facilitar el aprendizaje de los estudiantes de las áreas científicas y nos presentan metáforas como: “el genoma no es un plano para construir un cuerpo, es la receta para cocinar un cuerpo, lo que marca la diferencia es el tiempo de duración de una operación determinada" (Ridley, como se citó en Aguilar y Arroyo, 2008, p. 54) o "la mitocondria es una planta energética de la célula” (Lodish, como se citó en Aguilar y Arroyo, 2008, p. 54).

\section{La metáfora en la enseñanza}

Realmente, la metáfora es prolífica en el proceso de enseñanza y aprendizaje. Aunque hay algunas expresiones que se han vuelto tan recurrentes que no relucen ya como metáforas novedosas como: la claridad de un tema o las vías posibles para solucionar un conflicto, o el estudio de las distintas corrientes filosóficas; sí que siguen funcionando bien para nuestro entendimiento. Incluso, la comprensión del actuar pedagógico está repleto de metáforas.

Las corrientes del constructivismo llevan en su nombre una metáfora. El asunto de la construcción implica en ellas que el conocimiento es algo que se construye de manera activa por parte de los sujetos. Existen distintos enfoques sobre el constructivismo, Serrano y Pons (2011) los exponen como un continuo, y afirman que la galaxia en la que se mueven los posibles enfoques para la interpretación constructivista de los procesos de enseñanza y aprendizaje se puede ubicar en un continuo que sitúa la construcción del conocimiento en el sujeto individual, despreciando el componente socio-contextual de esa construcción (constructivismos endógenos); hasta posicionamientos que consideran el conocimiento social como la única fuente válida de conocimiento, con la consideración del sujeto colectivo como el elemento nuclear, negando, de esta manera, al sujeto individual (constructivismos exógenos).

En su afirmación, podemos notar la inclusión de una metáfora sideral cuando utilizan la figura de la galaxia para referirse a las posibilidades de movimiento que tienen las perspectivas constructivistas para observar a su objeto común de aprendizaje que es el sujeto conocedor. Aunque encontremos un universo de posibilidades de enfoques respecto a la teoría constructivista, sí 
que podemos reconocer algo en común y es que "[...] lo que las diversas formas de constructivismo comparten es la metáfora de la carpintería, la arquitectura y el trabajo de construcción. Esto se refiere a la construcción de estructuras a partir de piezas existentes" (Ernest, 1994, p. 1). Esto nos permitiría pensar sobre este concepto a partir de sus postulados comunes y reconocer si es la forma más práctica y efectiva de concebir el proceso de aprendizaje.

Existen, además, distintas teorías pedagógicas, por ejemplo, en la que se habla de la mente como un computador ${ }^{6}$, la ya en desuso idea del sujeto como una tábula rasa. Con toda seguridad, identificamos que estas perspectivas pedagógicas nombradas a través de metáforas no son un mero asunto de palabras, sino que realmente implican un cambio conceptual del proceso de aprendizaje y enseñanza, y modifican el rol que tiene el docente en él.

En las áreas específicas del conocimiento, también resultan diversas metáforas que ayudan a la comprensión del asunto. Por ejemplo, en la enseñanza de la técnica vocal suele ser común escuchar frases como: "Cantá con un sonido más blando y fluido", "Tratá de que la nota sea más puntiaguda y penetrante”, "Probá con un timbre más cálido, más redondo" (Vietri, 2017, p. 95). Estas expresiones se toman de la reseña que hace Maximiliano Vietri de un libro de Alessandronni, en la que dice que este último tiene como objetivo darle un valor a la metáfora en el ámbito pedagógico, atribuyéndoles el carácter de herramientas cognitivas y en el que "afirma que la metáfora, en la Pedagogía Vocal, implica no sólo un giro retórico, sino un recurso que propicia la comprensión por parte del estudiante" (Vietri, 2017, p. 95).

De igual manera, se pueden realizar actividades dentro de los espacios académicos que estén mediados por las metáforas. Por ejemplo, Pérez y Civarolo (2020) proponen invitar a

\section{[...] los estudiantes que se reúnan en comunidades de pensamiento e interactúen con fotografías de personas consideradas mentes brillantes, la actividad se acompaña con la rutina: creo-me problematizo-exploro, y se solicita que elaboren un texto utilizando una metáfora, en donde se explique y argumente qué es la inteligencia, qué significa ser inteligente hoy, y qué preguntas les genera el tema (p. 40).}

Desde este punto, es posible reconocer el valor cognitivo que tienen las metáforas, más allá de su carácter literario, estético y ambiguo que las ha hecho reconocidas como una torción del significado de las palabras. Así, este recurso tan reconocido

\footnotetext{
6 En términos estrictos, aquí se está utilizando un símil. Sin embargo, podemos reconocer la intención y el carácter metafórico de la expresión.
} 
en los campos literarios y que se presenta como un triunfo de la imaginación y del ingenio poético, demuestra que su poder llega a niveles más profundos del pensamiento humano y nos permite el aprendizaje de los nuevos conceptos que creamos en el mundo o de los nuevos descubrimientos que realizamos sobre la naturaleza de nuestro universo.

\section{La metáfora como herramienta cognitiva}

En la década de 1980, Lakoff \& Johnson plantean el término "Metáfora Conceptual" en un libro, cuyo título se tradujo como "Metáforas de la vida cotidiana”", para explicar este fenómeno, y argumentan que la metáfora es más que un recurso literario. Para ellos, la metáfora funciona como una herramienta cognitiva, cuya esencia es entender y experimentar una cosa en términos de otra, más que solo trasladar el nombre de un objeto a otro (p. 5). Aunque parezca que el cambio en la definición es mínimo, en la realidad tiene grandes implicaciones. Al afirmar esto, la metáfora se posiciona en el campo de las estructuras conceptuales y no en el del conocimiento lingüístico (Stöckl, 2010, p. 194). Así, es posible asir el concepto de metáfora como un asunto mucho más profundo de lo que aparenta ser y que se encuentra sedimentado en el fondo de las expresiones en las que aparece.

Esta perspectiva de la metáfora nace dentro del área de la lingüística cognitiva, que es “[...] un movimiento lingüístico que concibe el lenguaje como un fenómeno integrado dentro de las capacidades cognitivas humanas" (Ibarretxe y Valenzuela, 2012, p. 13). La preocupación por los aspectos cognitivos de los comportamientos humanos, entre ellos, uno tan complejo como el lenguaje, ha derivado en diversas ciencias que se encargan de estudiar partes específicas de ella y desde las que se apoya esta postura lingüística. Pero, observemos entonces, a qué nos referimos con las herramientas cognitivas.

\section{Herramientas cognitivas}

Iniciemos llamando la atención sobre la metáfora que conforma este subtítulo: Herramientas cognitivas. En términos literales, las herramientas son los instrumentos que utilizamos comúnmente para construir o reparar algo, como un martillo o una sierra, pero aquí, estamos utilizando ese nombre para hablar de las

\footnotetext{
7 El nombre del texto original es Metaphors we live by. Una traducción más literal, y a mi parecer mucho más congruente con su postulado, es "Metáforas a través de las que vivimos". Otra anotación es que la versión citada aquí es la edición del 2003.
} 
distintas formas en que funciona el pensamiento como si fueran instrumentos que utilizamos para ejecutar determinadas actividades cognitivas. Por supuesto, desde la postura de la metáfora conceptual, no solo hablamos de estas formas en términos de herramientas, sino que las comprendemos justamente como si lo fueran.

\section{Categorización}

Una de las herramientas que se considera de suma importancia para el postulado de la metáfora cognitiva o conceptual es la categorización, es decir, la inclusión que hacemos de objetos particulares a un grupo en nuestro entendimiento; la categoría de animales o plantas son ejemplos de esto. Así, para nosotros, un perro, un gato y un elefante pasan a formar parte de la categoría de animales, pero se excluye de esta a los árboles y a los lirios. Nuestra realidad cotidiana e intelectual está construida por categorías que surgen de nuestra experiencia e imaginación del mundo o estados posibles de este. Mientras nos movemos por él, automáticamente categorizamos personas, animales y objetos físicos, naturales y hechos por el hombre (Lakoff, 1987, p. 6).

La categorización "es una de las habilidades cognitivas básicas completamente imprescindibles para la supervivencia de cualquier sistema biológico" (Ibarretxe et al., 2012, p. 44). Las necesidades del organismo que realiza la categorización influyen en los atributos del mundo que va a percibir y a estructurar. Por lo tanto, las categorías en las que incluimos los diversos aspectos de la realidad estarían construidas, en primer lugar, por nuestra composición neuronal y fisiológica y, en segundo lugar, por la interacción de nuestro organismo con la realidad cultural.

Las categorías que conforman nuestra realidad conceptual están compuestas por objetos y entidades que funcionan directa o indirectamente en relación con nosotros: las enfermedades, los héroes, los buenos modales, los valores; son todos aspectos que consideramos como tipos de cosas a partir de nuestra interacción particular como humanos con el mundo físico y cultural, es decir, su inclusión en una categoría se da a partir de nuestra percepción y experiencia, y no de las propiedades naturales de cada uno de sus integrantes. Por ejemplo, categorizamos los planetas en un grupo de cuerpos celestes sin luz propia que orbitan en un sistema solar. Desde nuestra perspectiva, entendemos el sol como un punto central alrededor del cual gravitan tales cuerpos, pero estos cuerpos tienen propiedades distintas entre ellos, algunos son sólidos y otros gaseosos, y esto no representa un obstáculo para comprenderlos a todos como miembros de una categoría. Incluso, concebir al sol como un eje central del mundo en el que vivimos, depende absolutamente de nuestras condiciones biológicas y naturales propias. 
Rosch (1978), quien llevó la categorización al centro de atención como un problema fundamental de las ciencias cognitivas, encuentra que esta es producto de dos principios psicológicos. El primero es la economía cognitiva, que busca conocer la mayor cantidad de información del entorno con el menor gasto posible de recursos; Rosch plantea que, por un lado, es ventajoso para el organismo poder predecir la mayor cantidad de propiedades de un estímulo al conocer alguna de sus propiedades y, por el otro, que la categorización busca reducir la inmensa cantidad de diferencias entre los distintos estímulos a proporciones comportamentales y cognitivamente utilizables. El segundo, es la estructura percibida del mundo. Rosch nos dice que el mundo percibido no es una serie de atributos sin estructura que están coocurriendo, sino que este se percibe de manera tal que posea una estructura altamente correlacional. Por supuesto, esta estructura, estos atributos que se perciben, están determinados por las necesidades funcionales del conocedor para interactuar con el entorno físico y social (p. 4).

Así, la forma en que se estructura nuestro pensamiento, la manera en que ocurre eso que llamamos "pensar", se da a través de distintas herramientas cognitivas: por ejemplo, la abstracción, el razonamiento lógico, la identificación de unidades; la suma, la resta, entre otras; son herramientas del pensamiento que utilizamos normalmente y que nos ayudan a desenvolvernos en el mundo natural y social. Vimos que la categorización es una de ellas y a esta lista se añade la metáfora, que, en nuestros términos, es una traslación que ocurre en un nivel más profundo que el de las meras palabras y se descubre como la relación entre dos categorías del pensamiento o, como plantea la teoría de la metáfora conceptual, una relación entre dos dominios conceptuales.

\section{Integración conceptual}

Por definición, la metáfora menciona un tipo de cosas como si fuera otra. Desde el punto de vista cognitivo, esto implica que no solo se habla de una cosa en términos de otra, sino que realmente la comprendemos y experimentamos como si fuera tal. Esta comprensión no es enteramente la una o la otra, sino que, a través de la metáfora, surge una nueva idea a partir de los aspectos involucrados en el entendimiento de las dos.

La Integración Conceptual (CIT, por sus siglas en inglés) ${ }^{8}$ es una operación mental básica que conduce a nuevos significados, a una percepción global, y a compresiones conceptuales útiles para la manipulación de rangos de significado

8 Conceptual Integration Theory. 
difusos (Fauconnier \& Turner, 2003), a partir del emparejamiento de dos espacios mentales que proyectan algunos de sus aspectos en un nuevo espacio fusionado.

La noción fundamental de la CIT es que muchos pensamientos humanos consisten en la integración o fusión de espacios mentales, y la habilidad de realizar ciertos tipos de fusiones conceptuales es lo que distingue al humano de otros animales, y a la cognición humana moderna de las formas de cognición homínida anterior (Gill, 2010, p .25).

Fauconnier \& Turner plantean los siguientes puntos, los cuales fueron resumidos por Gill (2010, p. 25), y tienen implicaciones en la teoría de la Metáfora Conceptual:

- Espacios mentales: pequeños paquetes conceptuales que se construyen mientras pensamos y hablamos para la comprensión y acciones locales. Estos paquetes contienen la información del mundo que se va construyendo con las oraciones. Por ejemplo, en una oración como: ayer pasé por un lugar muy solo y oscuro, se genera un espacio mental que además de contener la información de la soledad y la oscuridad, también hay información no explicita sobre el camino que se menciona (si este es de piedra o de asfalto), sobre el lugar en que ocurrió (ciudad, vereda, pueblo), etcétera, que ayudan a la comprensión y comunicación efectiva.

- Espacios input: son espacios mentales usados como input, para la Integración Conceptual.

- Espacio genérico: este espacio se forma con lo que los espacios mentales tienen en común. Los elementos que se encuentran aquí se mapean a su contraparte en el espacio input, es decir, se hace una correspondencia uno a uno entre los elementos comunes de los espacios.

- Marcos: estructuras esquemáticas a largo plazo. Son las cosas que "ya sabemos”. Es en donde están conectados los espacios mentales.

- La integración o blend: también es un espacio mental, pero es el que se crea por la proyección de los espacios input. Esta integración es selectiva, es decir, no todos los elementos de los espacios input se proyectan en la integración.

- Estructura emergente: es la estructura que no está en los espacios input. Se genera a través de la composición (colocar elementos que no están en los espacios), la terminación (por ejemplo, completar un patrón que tiene elementos ausentes) o la elaboración ("correr" la simulación de la integración. Tratar la integración como una simulación y “correrla” por medio de la imaginación). 
La CIT plantea la unión de conceptos conocidos para la comprensión de un concepto nuevo. Un ejemplo que utilizan Fauconnier \& Turner (2003) para mostrar cómo funciona la Integración Conceptual es el de la "Carrera de Botes". Nos dicen que un Catamarán moderno navega desde San Francisco hasta Boston en 1993, intentado ir más rápido que un Clíper que navegó la misma ruta en 1853. Una revista de navegación reporta entonces: "Mientras íbamos a reportar, Rich Wilson y Bill Biewinga apenas mantenían una ventaja de 4.5 días sobre el fantasma de la embarcación Northern Light, cuyo récord de San Francisco a Boston, intentan vencer. En 1853, la embarcación realizó el recorrido en 76 días, 8 horas" (p. 58).

En este reporte hay dos eventos distintos: el recorrido que hizo el Clíper en 1853, y el recorrido del Catamarán en 1993, que se dan por, más o menos, el mismo curso. Estos dos eventos se mezclan en uno solo; la carrera entre el Catamarán y el "Fantasma" del Clíper. Cada uno de los dos eventos corresponde a un espacio mental. Los dos comparten un aspecto en común que es el recorrido. En este caso, el recorrido común constituye el espacio genérico. Luego tenemos la integración, que es la proyección selectiva de ambos espacios input. Es selectiva porque no se tienen en cuenta todos los aspectos que componen los eventos (aceleración de partida, estado emocional del conductor, clima del día, etcétera). Incluso, en el reporte nos mencionan solo tres aspectos: el tipo de embarcación, el recorrido y el tiempo en que se hicieron los recorridos. En este espacio integrado tenemos dos botes haciendo el mismo recorrido que salieron de San Francisco el mismo día. La estructura emergente nos permite completar el patrón y construir la situación como una carrera trayendo desde el marco de las "Carreras" las emociones que se asocian a ellas.

\section{La metáfora como la integración conceptual entre distintas categorías}

La metáfora cognitiva, que se define como entender y experimentar una cosa en términos de otra, implica justamente que hay una integración conceptual a partir de la activación simultánea de dos categorías conceptuales distintas. No funciona únicamente como la integración conceptual, pues esta se refiere a la integración de conceptos dentro de una misma categoría, como vimos en el ejemplo de la carrera de botes, y se apoya de la teoría de la categorización, ya que se evidencia una integración o unión conceptual de ideas que provienen de diferentes categorías.

En la teoría de la metáfora conceptual se nombra los conceptos que se unen como dominio fuente, que es el dominio desde el que se toman los conceptos que se usan para formar la metáfora, y el dominio meta, que es el concepto del que se está hablando metafóricamente. Si decimos, por ejemplo, que la mente 
es un computador, el dominio fuente sería el computador, y el dominio meta, la mente: puesto que estamos tomando la categoría de los computadores y lo relacionado con su funcionamiento, para referirnos metafóricamente al funcionamiento de la mente.

\section{Conclusión}

El lenguaje es un fenómeno que se encuentra integrado en las capacidades cognitivas humanas. Generalmente, se concibe a la metáfora como un recurso del idioma, una cuestión estilística y poética. Sin embargo, nos damos cuenta que las metáforas no se limitan al campo literario, también aparecen con gran profusión en el habla cotidiana. Además, evidenciamos que esta llega a niveles más profundos que el nivel semántico de la lengua, y la reconocemos como un recurso cognitivo que nos permite aprender nuevas cosas, percibir desde un enfoque distinto lo que conocíamos anteriormente, nombrar las nuevas cosas que vamos conociendo del mundo, y comprender los pormenores de las diferentes áreas de las ciencias y las artes humanas.

Identificamos pues la metáfora como una habilidad cognitiva a la par de la categorización y la integración conceptual, con elementos claros que demuestran su papel dentro de las habilidades mentales humanas como una herramienta inherente al proceso de aprendizaje.

\section{Referencias}

Aguilar, R., y Arroyo, A. (2008). La metáfora científica y los libros de texto. ContactoS, 67, 52-56. Ernest, P. (1994). Variedades de constructivismo: sus metáforas, epistemologías implicaciones pedagógicas. Hiroshima Journal of Mathematic Education, 2, 1-14. https://matematicaperu.files.wordpress.com/2012/12/equipo3_ernest1994_ constructivismo1.pdf.

Fauconnier, G., \& Turner, M. (2003). Conceptual blending, form and meaning. Recherches in communication, (19), 57-86.

Gill, T. (2010). Conceptual Blending, Metaphors, and the Construction of Meaning in Ice Age Europe: An Inquiry into the Viability of Applying Theories of Cognitive Science to Human History in Deep Time [Tesis de doctorado, Universidad de California, Berkeley]. https://escholarship.org/content/qt64x8g354/qt64x8g354_noSplash_ c13833a331442b2695523c0023bfeea7.pdf. 
Ibarretxe, I., y Valenzuela, J. (2012). Lingüística cognitiva: origen, principios teóricos y metodológicos, tendencias. En I. Ibarretxe y J. Valenzuela (Coords.), Lingüística cognitiva. Anthropos, 13-38.

Ibarretxe, I., Valenzuela, J., y Hilferty J. (2012). La semántica cognitiva. En I. Ibarretxe y J. Valenzuela (Coords.), Lingïística cognitiva. Anthropos, 41-68.

Lakoff, G. (1987). Woman, Fire, and Dangerous Things: What Categories Reveal About Mind. The University of Chicago Press.

Lakoff, G., \& Johnson, M. (2003). Metaphors we live by. The University of Chicago Press. https://nyshalong.com/public/archive/20150131/20150131_ref.pdf.

Pérez, M., y Civarolo, M. (2020). La metáfora como estrategia de enseñanza en el aula del siglo XXI. Revista chilena de pedagogía, 1(2), 25-48. https://revistadepedagogia. uchile.cl/index.php/RCHP/article/view/60555.

Restrepo, F. (1917). El alma de las palabras: diseño de semántica general. Editorial Barcelonesa.

Rosch, E. (1978). Principles of categorization. https://commonweb.unifr.ch/artsdean/pub/ gestens/f/as/files/4610/9778_083247.pdf.

Serrano, J., y Pons, R. (2011). El constructivismo hoy: enfoques constructivistas en educación. Revista electrónica de investigación educativa, 13(1), 1-27. https://www. redalyc.org/pdf/155/15519374001.pdf.

Stöckl, H. (2010). Metaphor Revisited: Cognitive-conceptual versus Traditional Linguistic Perspectives. Arbeiten aus Anglistik und Amerikanistik, 35(2), 189-207. http://www. periodicals.narr.de/index.php/aaa/article/download/542/520.

Vietri, M. (2017). Reseña de libro Las metáforas como herramientas cognitivas. Un acercamiento a su uso en pedagogía vocal. Epistemus, 5(1), 95-100. 


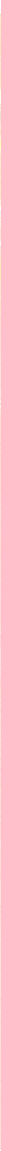

